\title{
Carnets
}

Revue électronique d'études françaises de l'APEF

Deuxième série - 14 | 2018

Études de génétique théâtrale et littéraire

\section{Traductions de théâtre ou trahison théâtrale : l'imitation renaissante comme appropriation culturelle}

Étude génétique du recueil des Six Premières Comédies facécieuses de Pierre de Larivey

\section{Anne Rogé}

\section{(2) OpenEdition}

\section{Journals}

Édition électronique

URL : http://journals.openedition.org/carnets/8607

DOI : $10.4000 /$ carnets.8607

ISSN : 1646-7698

Éditeur

APEF

Référence électronique

Anne Rogé, «Traductions de théâtre ou trahison théâtrale : l'imitation renaissante comme appropriation culturelle », Carnets [En ligne], Deuxième série - 14 | 2018, mis en ligne le 30 novembre 2018, consulté le 30 avril 2019. URL : http://journals.openedition.org/carnets/8607 ; DOI : 10.4000/ carnets.8607

Ce document a été généré automatiquement le 30 avril 2019.

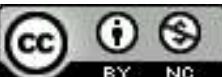

Carnets est mis à disposition selon les termes de la licence Creative Commons - Atribution - Pas d'utilisation commerciale 4.0 International. 


\section{Traductions de théâtre ou trahison théâtrale : l'imitation renaissante comme appropriation culturelle}

Étude génétique du recueil des Six Premières Comédies facécieuses de Pierre de Larivey

\section{Anne Rogé}

1 Traduttore, traditore. La paronomase italienne bien connue fait entendre plus clairement encore que l'expression française «Traduire, c'est trahir» le préjugé dénigrant la traduction. Si notre époque cherche à tout prix l'original, la source, la Renaissance se bâtit au contraire, comme son nom le suggère, sur l'idée d'un héritage, d'un renouveau : il s'agit pour les auteurs du XvI ${ }^{\mathrm{e}}$ siècle de faire renaître la littérature antique. Loin d'être perçue comme une "pâle copie » ou un plagiat, l'imitation représente alors un gage de qualité. En effet, l'imitation n'est ainsi pas perçue comme limitation, mais au contraire comme possibilité de mutation, de transformation d'une langue à l'autre. Dans le domaine de la comédie notamment, c'est d'abord la redécouverte des œuvres latines de Plaute en 1512, puis de Térence qui motivent la composition de comédies italiennes et françaises. Deux générations humanistes se succèdent ainsi en France : la première dans les années 1550 revalorise les œuvres antiques et encourage leur imitation dans la langue vernaculaire; la seconde, dans les années 1570, voit se développer un milieu plus italianisant, réinvestissant toujours les modèles antiques, mais à travers le relais des auteurs transalpins. Publiées en 1579 chez l'éditeur Abel l'Angelier, Les Six Premières Comédies facécieuses - à sçavoir Le Laquais, la Veuve, les Esprits, Le Morfondu, Les Jaloux, Les Escolliers de Pierre de Larivey appartiennent à cette seconde génération. Ce recueil comique se révèle emblématique de la problématique de l'imitation renaissante, puisqu'il met directement en relation la tradition humaniste italienne du début du $\mathrm{XVI}^{\mathrm{e}}$ siècle et son héritage français de la fin du siècle. L'étude de ces Comédies facécieuses nous confronte ainsi à l'épanouissement même du mouvement humaniste en Europe. Ce dernier, initié par l'Italie, se dynamise par le développement des échanges littéraires qui traversent alors les frontières européennes et dans le cas présent, celles des Alpes. 
2 L'ensemble du recueil comique de Larivey propose en effet une compilation organisée de six comédies reprises et traduites de l'italien. Il est intéressant de remarquer que chacune est composée par un auteur italien différent, mais qu'elles relèvent pourtant toutes du genre de la commedia erudita - c'est-à-dire de la comédie érudite ou régulière. Le catalogue établi par Michel Simonin et Jean Balsamo énumère avec précision les auteurs des six comédies italiennes. Nous en reproduisons ici la liste : «Lodovico Dolce (Il Ragazzo, 1541), Niccolo Buonaparte (La Vedova, 1568), Lorenzino de' Medici (L'Aridosia, 1549), Anton Francesco Grazzini dit Il Lasca (La Gelosia, 1551), Vincenzo Gabbiani (I Gelosi, 1557), Girolamo Razzi (La Cecca, 1563) ». (Simonin et Balsamo, 2002 : 158.). La lecture de ces titres laisse déjà entrevoir la gamme de personnages sélectionnée par Larivey, suggérant les tensions propres au genre de la comédie érudite. Laquais, veuve, avare, jaloux: ces dénominations créent un horizon d'attente pour le lecteur, mettant en scène des conflits sociaux - entre maîtres et valets -, familiaux - entre jeunes et vieillards -, mais aussi sentimentaux. L'enjeu de notre recherche réside dans l'appréhension de ces comédies comme un ensemble organisé et agencé par les soins de Larivey.

Notre étude s'efforce de rendre compte de la complexité de l'imitation renaissante au sein de l'œuvre de Larivey. Cette approche se fonde sur la mise en réseau des six comédies avec les textes liminaires qui ouvrent le recueil. Ouvrir cette communication à travers l'œuvre permet de mettre en valeur un paradoxe fondamental de la genèse du recueil pour le lecteur d'aujourd'hui. En effet, alors que Pierre de Larivey insiste sur le caractère nouveau de son ouvrage dans l'épître dédicatoire, il reconnaît dans le même temps travailler «à l'imitation des anciens Grecs et Latins et modernes Italiens. » Ce double mouvement d'hésitation entre l'imitation et l'innovation, nous invite finalement à nous demander de quelle façon la composition des Six Premières Comédies facécieuses de Pierre de Larivey, toutes imitées de l'italien, constitue une innovation littéraire en France.

\section{L'imitation comme système génétique moderne}

4 Il s'agit dans un premier temps de considérer ce que signifie écrire "à l'imitation des anciens Grecs, Latins et modernes Italiens " pour Pierre de Larivey, comme il l'annonce dès le sous-titre de son ouvrage. Contrairement aux idées reçues, imiter les ouvrages antiques au $\mathrm{XVI}^{\mathrm{e}}$ siècle relève d'un engagement littéraire "moderne", initié par le mouvement de la Pléiade. Lorsqu'elle émerge au milieu du siècle, la Renaissance est un mouvement controversé : d'une part, les jeunes poètes de la Pléiade affirment le besoin de développer une littérature qui soit à la hauteur des œuvres antiques, tandis que d'autre part, certains auteurs défendent la tradition médiévale française. Pour Joachim du Bellay, les œuvres médiévales sont jugées trop vulgaires et incapables de soutenir la gloire française, comme il l'exprime dans le second livre de La Deffence et Illustration de la Langue Françoyse :

Quant aux comédies et tragédies, si les rois et les républiques les voulaient restituer en leur ancienne dignité, qu'ont usurpée les farces et moralités, je serais bien d'opinion que tu t'y employasses, et si tu le veux faire pour l'ornement de ta langue, tu sais où tu en dois trouver les archétypes. (Du Bellay, 1549 : livre II, chap. 4).

La pratique de l'imitation au $\mathrm{XVI}^{\mathrm{e}}$ siècle se fonde sur un rapport volontaire entre un texte existant et un texte en cours de création. Les modèles choisis diffèrent cependant d'une génération humaniste à l'autre: tandis que vers 1550, ils sont avant tout issus de l'Antiquité, à partir de 1570, les œuvres italiennes s'affirment comme modèles 
vernaculaires, égalant la qualité des Anciens. Madeleine Lazard rappelle cependant à juste titre que :

L'admiration pour les modèles de l'Antiquité et de la France n'exclut pas le désir de rivaliser avec eux et même de les surpasser. Jodelle conseille le premier de « plus en plus ozer bien davantage », La Taille affirme que «nostre langue pour le présent n'est en rien inférieure à la leur » et François d'Amboise, en 1584, estime que la France a "surpassé les Itales» dans le domaine tragique et comique. (Lazard, 1980 : 167.)

Ce phénomène d'imitation représente ainsi un gage d'émulation créatrice, tant pour les auteurs de la Pléiade, comme Étienne Jodelle et Jean de La Taille, que pour les auteurs de la seconde génération humaniste, tels que François d'Amboise, Odet de Turnèbe et Guillaume Le Breton. Respectivement auteurs des Napolitaines, des Contents et de plusieurs tragédies humanistes imitées et inspirées d'auteurs transalpins, ces trois dramaturges constituent avec Pierre de Larivey le noyau même du milieu parisien italianisant. Tous trois représentent d'ailleurs les interlocuteurs privilégiés des textes liminaires du recueil de notre auteur. En effet, le sonnet d'hommage dédié « au Seigneur de L'Arrivey » porte la devise de Guillaume Le Breton, tandis que Pierre de Larivey adresse lui-même son épître dédicatoire à François d'Amboise, et termine sur ces mots :

Et ce sont les raisons desquelles vous et Monsieur le Breton, que j'honore beaucoup pour ses rares vertus, m'avez plus eguillonné de donner commencement à ces fables, qu'icy je vous offre et desdie, comme au meilleur de mes meilleurs amis, affin que vous, qui estes mon auteur et garand formel, preniez, s'il vous plaist, la cause pour moy [...] (Larivey, 1855 : 3.)

7 L'ouvrage de Pierre de Larivey émerge ainsi au sein de ce milieu marqué par l'imitation d'œuvres italiennes. L'ensemble de l'épître vise d'ailleurs à expliquer son projet comique " nouveau ", au sens où il n'y a pas d'antécédent en langue française. Toute la valeur de l'imitation se réalise en effet dans le développement fructueux d'une langue et d'une littérature nationales.

Les jeux d'influence à l'œuvre, du grec au latin, du latin à l'italien, de l'italien au français, contribuent à un amalgame de sources se révélant toujours innovant. Prenons l'exemple de la troisième comédie du recueil de Larivey : Les Esprits. Dans cette pièce, trois intrigues amoureuses se tissent, toutes trois soumises aux décisions paternelles de deux frères aux méthodes d'éducation opposées : Hilaire et Séverin. En remontant aux modèles de cette comédie, nous découvrons la richesse de son héritage. Si la pièce des Esprits reprend fidèlement la trame italienne de L'Aridosia de Lorenzino de Medici, elle conserve tout de même l'opposition de deux frères, inspirée des Adelphes de Térence ainsi que la figure de l'avare, issue de L'Aulularia de Plaute. Lorsque Pierre de Larivey choisit la comédie italienne, il imite en conscience une pièce en langue vernaculaire, mais aussi ses patrons antiques. Passer de l'italien en français ne constitue donc pas une simple reprise, mais aussi - et même avant tout - un travail d'appropriation d'une grandeur nationale. En effet, au $\mathrm{XVI}^{\mathrm{e}}$ siècle, l'imitation est motivée par les concepts de translatio imperii et translatio studii, c'est-à-dire l'idée que la gloire politique et littéraire se transmettent au fil du temps, de nation en nation. Dans la continuité de la Grèce antique, de l'Empire romain et de l'épanouissement renaissant en Italie, les auteurs français attendent et espèrent que cette prospérité se révèle dans leurs œuvres. La pensée de cet héritage éveille la conscience de la valeur de la langue comme expression d'un rayonnement intellectuel. Il s'agit là un élément fondamental à la différenciation de la traduction et l'imitation : alors 
que le processus de traduction met deux œuvres en parallèle, l'imitation cherche à dépasser le modèle pour l'effacer et s'imposer à sa place.

Il faut dès lors prendre en considération les parts respectives de l'auteur-modèle et celle de l'auteur-imitateur. Il y a dans le cas de l'œuvre comique de Pierre de Larivey un élément essentiel à ne pas négliger : l'imitateur est unique tandis que ses modèles sont divers. L'imitation de Larivey fonctionne donc non seulement comme un phénomène de reprise mais aussi comme un phénomène d'unification: à partir de six pièces italiennes composées par six auteurs différents, Larivey constitue une œuvre unique, en langue française. Ce processus d'unification est d'ailleurs souligné par Jean Balsamo lorsqu'il écrit que «Larivey proposait ainsi l'œuvre comique la plus consistante et la plus homogène de son temps, et il la tirait d'une utilisation systématique des Italiens " (Balsamo, 1992 : 272). Le rapport entre le modèle et l'imitateur fonctionne en effet pour Larivey à la façon d'un "patron" qu'il utiliserait pour guider sa composition. Cette métaphore du tissage évoquée par Larivey lui-même dans son épître est essentielle. En effet, elle se révèle définitoire du degré de similitude entretenu entre les textes italiens et français. Larivey reprend systématiquement la structure des comédies érudites italiennes. Cela confère une régularité à l'ensemble du recueil puisque chaque comédie compte cinq actes divisés en scènes, une intrigue clairement structurée, mais aussi une galerie homogène de personnages. S'il conserve ainsi l'architecture globale des pièces, Pierre de Larivey s'efforce cependant d'apporter plus de naturel et de dynamisme aux pièces françaises. Il recherche davantage de simplicité dans le déroulement de l'action. Nous pouvons d'ailleurs observer à cet égard une réduction importante du nombre de scènes par actes que résume le tableau qui suit :

\begin{tabular}{|c|l|}
\hline \multicolumn{2}{|l|}{ Nombre de scènes par comédie } \\
\hline Comédies italiennes & Comédies françaises \\
\hline Il Ragazzo -45 scènes & Le Laquais -25 scènes \\
\hline La Vedova - 53 scènes & La Veuve -37 scènes \\
\hline L'Aridosia -33 scènes & Les Esprits -30 scènes \\
\hline La Gelosia -51 scènes & Le Morfondu - 32 scènes \\
\hline I Gelosi - 40 scènes & Les Jaloux - 30 scènes \\
\hline La Cecca - 31 scènes & Les Escolliers - 29 scènes \\
\hline
\end{tabular}

Dans sa recherche d'efficacité dramatique, Pierre de Larivey élimine donc de nombreuses scènes, mais aussi des personnages. À l'exception de la pièce des Escolliers qui comporte 16 
personnages dans les deux versions, toutes les comédies du dramaturge français comptent moins de personnages que les pièces italiennes, comme le montre le tableau suivant :

\begin{tabular}{|l|l|}
\hline \multicolumn{2}{|l|}{ Nombre de personnages par comédie } \\
\hline Comédies italiennes & Comédies françaises \\
\hline Il Ragazzo - 13 personnages & Le Laquais - 12 personnages \\
\hline La Vedova - 20 personnages & La Veuve - 15 personnages \\
\hline L'Aridosia - 17 personnages & Les Esprits - 12 personnages \\
\hline La Gelosia - 15 personnages & Le Morfondu - 13 personnages \\
\hline I Gelosi - 21 personnages & Les Jaloux - 17 personnages \\
\hline La Cecca - 16 personnages & Les Escolliers - 16 personnages \\
\hline
\end{tabular}

11 Garder à l'esprit ce travail de coupe effectué par Larivey permet de mieux appréhender les contraintes scéniques qu'il essaie de respecter. En effet, comme le remarque le libraire Pierre Jannet dans son avertissement, "[Larivey] supprimoit des scènes et des rôles, surtout des rôles de femmes; il ajoutoit rarement » (Larivey, 1855 : 16). Il ne s'agit donc pas seulement de condenser l'intrigue, mais aussi de tenir compte des nécessités de la mise en scène : il est alors difficile de faire jouer des jeunes femmes sur le théâtre. Larivey compose ainsi ses comédies en imitateur averti: il s'inscrit dans un mouvement humaniste moderne, visant à restaurer le modèle régulier de la comédie antique relayé par les auteurs renaissants italiens et est conscient des contraintes de la scène française. Le poids de l'imitation préside à la genèse de l'œuvre comique de Pierre de Larivey puisqu'il prend soin d'expliquer sa démarche au sein des textes liminaires. Un premier élément de réponse apparaît dès lors pour expliquer le paradoxe de la nouveauté de son travail d'imitation: Larivey fait œuvre nouvelle dans le champ du théâtre en langue française par la modernité dont il fait preuve, tout comme les Italiens l'ont fait avant lui. Son ouvrage est "basty à la moderne", au sens où il suit le schéma structurel des comédies italiennes. 


\section{De la traduction à la naturalisation française des pièces italiennes}

12 Si les marques de l'imitation apparaissent d'un point de vue structurel, la langue même des textes laisse davantage voir le profond travail de traduction mis en œuvre par Larivey. Il s'agit ainsi dans un deuxième temps de s'attarder sur l'ambiguïté des limites entre traduction et imitation, mises à l'épreuve dans les six comédies de Larivey. La frontière entre imitation et traduction est en effet particulièrement subtile dans les Six Premières Comédies facécieuses. Comme l'exprime Madeleine Lazard dans son article intitulé «Les Belles infidèles de Pierre de Larivey »:

[L]a critique a souvent fait grief à Larivey du vague prudent qu'il a laissé planer sur ses sources. Il transpose si adroitement ses modèles qu'il ne donne jamais l'impression de traduire. Aussi a-t-il pu passer longtemps pour un auteur original [pourtant] ce sont les «patrons » italiens qu'il imite. Il va donc se trouver à leur égard dans le même rapport de dépendance, sans différencier très clairement, comme presque tous les théoriciens de l'époque, l'activité d'imitation de celle du traducteur. (Fragonard, $2000: 78$.)

13 La majorité des répliques des six comédies est en effet traduite littéralement des textes italiens : Pierre de Larivey ne s'éloigne de la traduction littérale que dans des cas bien délimités. Cet usage parcimonieux transparaît surtout pour transformer les références italiennes en françaises. Il suit à cet égard le précepte qu'il annonce dans son épître, selon lequel les scènes italiennes doivent être adaptées à la réalité française de son temps :

J'ay dict que j'en jette les premiers fondemens, non que par là je veulle inferer que je sois le premier qui faict des Comedies en prose, car je sçay qu'assez de bons ouvriers, et qui meritent beaucoup pour la promptitude de leur esprit, en ont traduict quelques unes; mais aussi puis-je dire cecy sans arrogance, que je n'en ay encores vu de françoises, j'enten qui ayent esté representées comme advenues en France. (Larivey, $1579: 2$ )

14 Les références topographiques et culturelles se trouvent ainsi, non pas traduites de l'italien au français pour donner une couleur italienne aux comédies comme Larivey le fait dans Les Facécieuses Nuicts de Straparola, mais au contraire annexées aux représentations communes françaises: il s'agit en traductologie de naturalisation. Pour reprendre l'expression de Luigia Zilli, « Naturaliser une intrigue signifie - pour Larivey l'ancrer aux références géographiques, historiques, culturelles, sociales et même culinaires de son pays et de son temps. » (Larivey, 2011:21). Pierre de Larivey naturalise ses traductions pour leur conférer un caractère d'authenticité française. C'est bel et bien ce système d'équivalence des représentations partagées que notre dramaturge développe au sein de son recueil de comédies. Il s'évertue en effet à leur donner une couleur locale, inspirée du milieu parisien où il évolue. Il y a de la part de Larivey un profond travail de compréhension des langues comme prismes d'une vision du monde. Il n'entreprend pas seulement de traduire des noms de lieux étrangers, mais il s'efforce de faire correspondre deux réalités culturelles. Prenons deux exemples représentatifs du travail de Larivey: le système d'équivalence monétaire et la naturalisation des références littéraires. L'argent étant au cœur de la pièce des Esprits avec l'avarice de Séverin, Larivey exploite délibérément le vocabulaire pécuniaire pour faciliter la transposition sur la scène française. Adaptant le texte de L'Aridosia à celui des Esprits, Pierre de Larivey porte ainsi un soin particulier à convertir l'ensemble des monnaies italiennes évoquées en leur 
équivalent français respectif: les «soldi ", «scudi » et «ducati » sont remplacés par les «liards", les "francs» et les «livres». Outre cet exemple issu des représentations communes de la vie quotidienne, Larivey naturalise également le socle de références intellectuelles et notamment littéraires. La comédie inspirée du Ragazzo de Lodovico Dolce notamment propose des évocations des célèbres poètes italiens contemporains, tels que Pietro Bembo. Pierre de Larivey s'applique à remplacer les références transalpines par celles de leurs équivalents français, à savoir Pierre de Ronsard et Jean-Antoine du Baïf, comme le montre cet extrait de la réplique du personnage de Thomas: «Et puis dictes que je n'y enten rien! Suis-je pas bon poëte? Oy, par Dieu, et si jamais je ne me suis alambiqué le cerveau à lire en Ronsard, Baif, et autres qui composent à leur mode, et moy à la mienne.» Le texte italien fait dire au personnage de Ciacco: "Non sono io buon poeta ? Sì, sono, per Dio. Senza ch'io m'abbia beccato il cervello in lettere, io la'mpaterrei al Bembo ch'è, non pur poeta, ma cardinale. Ma rime a sua posta. » La naturalisation effectuée par Larivey rend la référence beaucoup plus significative pour les spectateurs français. En outre, le fait de remplacer Pietro Bembo par Pierre de Ronsard est suggestif de l'état des connaissances littéraires de Pierre de Larivey : il est tout à fait familier des références tant françaises qu'italiennes qui irriguent la littérature de son temps. En auteur averti, il lui est facile de substituer ces deux auteurs marqués par l'inspiration de Pétrarque. Ces exemples issus des première et troisième comédies du recueil donnent à voir et entendre le ton naturel que recherche Pierre de Larivey : celui de la langue et de la littérature françaises, qui ose s'exprimer en prose. C'est là que réside l'un des engagements modernes de Larivey, encore controversé à l'époque, si l'on en juge par l'explication qu'il donne dans l'épître dédicatoire :

Or, si je n'ay voulu en ce peu, contre l'opinion de beaucoup, obliger la franchise de ma liberté de parler à la severité de la loy de ces critiques qui veullent que la Comedie soit un poëme subject au nombre et mesure des vers (ce que, sans me vanter, j'eusse pu faire), je l'ay faict parce qu'il m'a semblé que le commun peuple, qui est le principal personnage de la scène, ne s'estudie tant à agencer ses paroles qu'à publier son affection, qu'il a plutost dicte que pensée. (Larivey, 1579 : 2.)

Cette recherche de proximité avec le naturel de l'expression populaire porte la nouveauté et le caractère moderne de l'ouvrage de Larivey. En naturalisant les références italiennes des comédies, il enrichit la littérature française de textes nouveaux. Larivey poursuit ici l'œuvre dubellayenne, puisqu'il offre en France une illustration en prose des préceptes défendus dans La Deffence et Illustration de la langue française. Il illustre la langue de son pays, c'est-à-dire qu'à la fois il la développe, valorise et met en lumière par le recueil de comédies qu'il publie. Ce n'est plus tant l'heure du combat auquel invite Du Bellay en 1549, mais bien plutôt l'heure de l'épanouissement de la littérature française. Comme le résume Antoine Berman, la traduction « est pour les écrivains de cette époque le lieu où l'on forme sa langue ", où on lui donne forme par l'élargissement de son vocabulaire, que l'on s'approprie de l'étranger (Berman, 2012 : 169). La profonde nouveauté ne réside pas dans les comédies mêmes, mais dans la langue au sein de laquelle elles s'écrivent.

\section{米*}

16 Si Pierre de Larivey défend depuis sa dédicace le bien-fondé d'une écriture comique en prose, il semble pourtant prendre plaisir à rechercher les rimes de la poésie lorsque l'occasion se présente dans ses modèles italiens. Les pièces du Laquais, de La Veuve et des Esprits en proposent des exemples particulièrement intéressants dans le cadre de notre 
étude. Toutes les éditions distinguent d'ailleurs ces moments poétiques par le style d'écriture utilisé, recourant généralement à une taille de police plus petite et aux caractères en italiques. Dans ces trois comédies, Larivey développe ou transforme un court passage rimé, voire un extrait en prose en un poème plus ou moins long. Dans Le Laquais, le personnage du proxénète Thomas entonne tout à coup une chanson paillarde :

Mes dames si quelque testu

Veut croire qu'amour soit vertu,

Il est sans yeux, \& ne void goute,

Et va errant par un chemin,

Qui le detournant de sa route

Le faict egarer à la fin.

Sçavez vous qui est nostre mieux,

Et qui nous faict egaux aux Dieux?

C'est d'estre content en son ame,

Et jamais rien ne desirer,

Car le desir est une flamme,

Qui ne nous faict que martirer.

承

D'Amour tous debats sont venus,

Et les biens viennent de Venus,

Et de ce bon Dieu de la treille,

C'est pourquoy tousjours de bon coeur,

Je sacrifie la bouteille,

Et la pucelle à leur honneur. (Larivey, $1855: 33$.)

Dans La Veuve, c'est le personnage de l'entremetteuse Guillemette qui fait l'éloge d'une bouteille de vin, rappelant le genre des chansons à boire : « Ma bouteille, si la saveur / De ce vin respond à l'odeur, Je prie Dieu et Saincte Heleine / Qu'ils te maintiennent tousjours plaine.» (Larivey, 1855 : 133.). Dans la comédie des Esprits enfin, le prêtre de la version italienne devient un sorcier déclamant des imprécations pour chasser les esprits éponymes de la maison de l'avare, dont voici un extrait: "Esprits maudits des infernalles ombres, / Qui repaiez ceans soir \& matin, / Je vous commande au nom de Severin, / Qu'en deslogiez sans nous donner encombres. » (Larivey, $1855: 242$.) Observer successivement ces trois situations permet de mettre en relief l'usage de formes versifiées dans les moments de tension des comédies, toujours attribuées aux personnages malveillants. Le caractère répréhensible de leurs actes trouve à chaque fois une forme d'expression versifiée. Le décalage entre leur comportement et l'inspiration poétique qui les caractérise est propice à susciter le rire du public, mais aussi à lui faire comprendre la critique moralisatrice sous-jacente. Comme l'écrit Patrizia de Capitani, «Larivey a voulu apporter des éléments (personnages, intrigues, situations comiques, rapports entre comédie et réel) susceptibles d'enrichir la scène française.» (De Capitani, 2005 : 247). La complexité des rapports entre traduction et adaptation rejaillit ainsi au sein du processus de l'imitation. Cuvrer à la frontière des textes littéraires incite Pierre de Larivey à naturaliser les comédies en France, quitte parfois à ajouter des inventions de son cru. Ce dépaysement des six pièces de théâtre confère ainsi une couleur locale parisienne à l'ensemble du recueil. Ce dernier s'affirme en effet comme relais français des littératures antiques et italienne au sein duquel Larivey développe progressivement un système abouti de composition dramaturgique. 


\section{Un système d'imitation bien rodé par la mise en recueil}

17 Il s'agit dans ce troisième temps de rendre compte de l'impact de la publication de ces six comédies sous la forme d'un recueil. La sélection réalisée est en effet le fruit du travail de Pierre de Larivey. En rassemblant ces diverses comédies érudites et en les offrant au public, traduites en français, unifiées dans une langue qui efface les disparités linguistiques des régions italiennes, ce recueil réalise l'une des ambitions de Joachim du Bellay : il restaure le genre antique de la comédie en France. Par son travail de reprise structurelle et de naturalisation linguistique, le recueil réalise l'appropriation culturelle du genre comique. D'ailleurs, comme le signale Guy Degen,

le choix [de Larivey] ne s'était pas porté sur des œuvres d'auteurs célèbres, déjà considérées comme des chefs-d'œuvre. Larivey aurait pu en trouver la première anthologie dans le Libro primo delle commedie elette de G. Ruscelli, paru dès 1554. Mais ces œuvres-là, les connaisseurs les avaient déjà lues dans leur langue, et leur traduction, surtout déguisée, ne les aurait ni intéressés ni trompés. Aussi Larivey, par coquetterie de découvreur autant que par ruse, avait-il choisi trois des comédies qui sont restées, dans le genre, des filles uniques de leur auteur: La Vedova de N. Buonaparte (1568), L'Aridosia de Lorenzino de' Medici (1548) et I Gelosi de V. Gabiani (1551) (Bellenger, 1993 : 16).

18 Ce choix d'œuvres peu célèbres en France permet à Larivey de présenter ces comédies éparses sous un nom unique, le sien. Le statut de « découvreur » suggéré par Guy Degen signale une nouvelle particularité du travail de Pierre de Larivey. Imiter, réécrire, c'est à la fois découvrir et faire découvrir. La connaissance de la langue italienne par Pierre de Larivey lui permet d'être ce découvreur, cet explorateur littéraire pour ses compatriotes. Il propose un corpus d'auteurs représentatif de la diversité des comédies érudites. En effet, les origines régionales varient suivant les auteurs, originaires de Venise à Florence. De plus, l'écart temporel qui sépare la publication des pièces s'étale sur plus de vingt-cinq ans, depuis Il Ragazzo en 1541 jusqu'à La Vedova en 1568. L'ordre chronologique retrace donc un autre ordonnancement des pièces sélectionnées par Larivey. Le phénomène de découverte consiste dans cette ruse qui lui permet de revendiquer paradoxalement le mérite de la nouveauté en tant qu'imitateur. Si le "patron" des modèles italiens est succinctement annoncé dans la préface, c'est avant tout un recueil en français que notre auteur tient à présenter.

19 Le recueil s'impose ainsi comme facteur d'affirmation du genre comique. Nous avons pu observer jusqu'à présent que Pierre de Larivey entreprend un travail important en publiant le premier recueil théâtral en français, composé exclusivement de comédies. Le caractère novateur de cette entreprise réside dans la définition d'un genre nouveau en France : la comédie régulière. Le travail de sélection, de recomposition et d'agencement des six comédies élabore une approche complètement renouvelée des textes originaux. La logique d'un lire ensemble transcende ainsi la lecture et la représentation ponctuelle de comédies. Ce dépassement du statut habituel du texte théâtral s'opère en quelque sorte par la recherche de ce qui définit la comédie en soi. La confrontation à un corpus organisé offre l'occasion de s'éloigner d'une lecture littérale au profit d'une approche critique des différents textes. Cette conscience réflexive de la valeur de chaque comédie en elle-même et en rapport aux autres constitue l'élément fondateur d'une pensée générique. Le genre est en effet le dépassement du particulier vers le général. Il permet de classer les 
différentes productions littéraires selon des traits généraux. Cet usage du vocable de " genre " pour évoquer une catégorie d'œuvres définies par la tradition littéraire apparaît en 1549. Sujet, ton et style déterminent ainsi un genre particulier. Quand Pierre de Larivey s'empare du genre comique, ce sont ces trois caractéristiques qu'il entreprend de définir. Pourtant, si le terme de genre apparaît trente ans avant sa publication, nous pouvons nous demander si Pierre de Larivey définit ou illustre le genre comique. À cet égard, la remarque d'Irène Langlet sur la dynamique qui anime les notions de genre et de recueil se révèle fondamentale :

La liaison du recueil au genre des textes qu'il rassemble oriente encore nettement la réflexion [...] Peut-être parce que le recueil représente un cobaye inespéré, une sorte de condensé de toute dynamique du genre: le rapport de chaque unité à l'archétype formel, dans le cadre d'une série (ouverte ou close) où l'interaction du tout et de la partie emprunte, avec d'infinies variétés, deux grands itinéraires possibles: le genre peut faire le recueil, ou bien le recueil peut faire le genre. (Langlet, $2003: 14$.)

Si l'on suit cette perspective suggérée par Irène Langlet, l'ouvrage de Pierre de Larivey semble bien avoir pour fonction d'étayer la pensée d'un genre. Le recueil de 1579 ne crée pas la notion de genre comique mais il l'expérimente et la fait advenir par la composition dramatique. À cet égard, l'œuvre de Larivey vient illustrer le manifeste de la génération précédente écrit par Joachim du Bellay. Par la publication de son recueil, Pierre de Larivey participe non seulement au développement d'une littérature en langue française, mais aussi à l'émergence d'un genre littéraire. L'édition inaugurale d'un recueil théâtral a le mérite d'éveiller la conscience d'une littérature organisée en diverses catégories, guidées par des aspirations différentes. L'apport de Pierre de Larivey est ainsi donc multiple : il augmente de façon conséquente la production comique du siècle, contribue par la publication en recueil à l'émergence d'une conscience générique et profite également de la flexibilité formelle du recueil pour dresser un état des lieux de la comédie.

\section{Conclusion}

$\mathrm{Au}$ terme de cette étude, nous devons noter combien la perception de l'imitation à la Renaissance est différente de celle que nous en avons aujourd'hui. Ni pure traduction, ni trahison honteuse de ses modèles, l'imitation représente au $\mathrm{XvI}^{\mathrm{e}}$ siècle l'un des modes d'écriture les plus répandus et les plus estimés en France. Loin d'être perçu comme une dette contractée envers les auteurs italiens, l'aveu de Larivey de travailler à l'imitation des auteurs antiques et italiens accrédite la valeur de son ouvrage puisqu'il montre qu'il est parvenu à introduire en France la qualité des grandes œuvres littéraires étrangères. Nous avons pu observer combien les préceptes de la première génération humaniste innervent l'œuvre de Larivey. Il lui faut s'inspirer des grandes littératures pour constituer la sienne. Dans la continuité de la Pléiade, il s'efforce donc d'illustrer la littérature de son pays par de nouveaux exemples empruntés de l'autre côté des Alpes. C'est l'apport dans la littérature française qui consacre le caractère "nouveau» des comédies: Pierre de Larivey effectue un travail dans et pour la langue française. En faisant passer les textes d'une comédie d'une langue à une autre, il les importe au sein de sa langue. Le travail conjoint de la traduction et de la naturalisation des références nationales confère aux six comédies le naturel de la couleur locale. Ce processus d'appropriation culturelle participe au développement de la langue nationale. Nous avons vu que le travail de traduction fait 
partie intégrante de l'imitation au $\mathrm{XVI}^{\mathrm{e}}$ siècle. C'est justement dans la recherche du mot juste, de l'expression adéquate que Larivey forge sa langue. La relation du modèle à l'imitateur est par essence constructrice : il s'agit de bâtir une langue vernaculaire riche.

Comme nous l'avons observé, son travail va au-delà de l'imitation formelle et de la naturalisation linguistique puisqu'il suit une logique d'affirmation de la comédie. Nous avons exprimé au sein de notre étude la logique de l'association des comédies par l'expression d'un lire ensemble. Réunir cet ensemble de comédies fait émerger une systématisation dans l'esprit du lecteur. Si Pierre de Larivey importe des comédies à partir d'exemples italiens, il les bâtit au sein d'un édifice plus solide, organisé de façon méthodique autour de chaque pièce. La composition en recueil a un impact significatif sur la réception des comédies. Il se dégage en effet une unité débordant l'écriture comique proprement dite. Recueillir au sein d'un même volume des pièces diverses permet de prendre conscience de l'existence d'un mouvement littéraire. La sélection fait toute la singularité de l'œuvre puisqu'elle vient illustrer une logique générique: celle de la comédie régulière. Un mouvement de va-et-vient s'instaure entre l'expérimentation du genre et sa définition dans l'épître dédicatoire, comme si Larivey souhaitait que ses six comédies servent d'exemples pour illustrer sa pensée de la comédie. C'est donc bel et bien un relais français du genre comique en langue française que Pierre de Larivey met en place. Le fait même que Molière puise moins de cent ans après dans les répliques de Séverin pour composer le personnage d'Harpagon est représentatif des ressources comiques que Larivey a offertes à la scène française.

\section{BIBLIOGRAPHIE}

BALSAMO, Jean (1992). Les Rencontres des muses : italianisme et anti-italianisme dans les lettres françaises de la fin du XVI ${ }^{e}$ siècle. Genève-Paris : Slatkine, «Bibliothèque Franco Simone », nº 19.

BALSAMO, Jean et SIMONIN, Michel (2002). Abel L'Angelier et Françoise de Louvain (1574-1620). Suivi du Catalogue des ouvrages publiés par Abel L'Angelier (1574-1610) et la Veuve L'Angelier (1610-1620). Genève : Droz, « Travaux d'humanisme et Renaissance », $n^{\circ} 358$.

BELLENGER, Yvonne (dir.) (1993). Pierre de Larivey (1541-1619). Champenois, chanoine, traducteur, auteur de comédies et astrologue, actes des sixièmes journées rémoises et troyennes, 25-27 janvier 1991, organisées par le Centre de Recherche sur la Littérature du Moyen Âge et de la Renaissance de l'Université de Reims. Paris : Klincksieck, « Actes et colloques », $\mathrm{n}^{\circ} 36$.

BERMAN, Antoine (2012). Jacques Amyot, traducteur français. Essai sur les origines de la traduction en France. Paris : Belin, « L'Extrême contemporain ».

BUONAPARTE, Niccolò (2003). La Vedova, comedia facetissiama di M. Nicolo Buonaparte, cittadino fiorentino [1568]. Pise : ETS.

CAPITANI, Patrizia de (2005). Du spectaculaire à l'intime. Un siècle de commedia erudita en Italie et en France (début du XVI ${ }^{e}$ siècle - milieu du XVII siècle). Paris : Honoré Champion. 
DOLCE, Lodovico, Il Ragazzo (1925), dans : Ireneo Sanesi, Commedie del Cinquecento, t. II, G.Laterza, Bari, « Scrittori d'Italia », $\mathrm{n}^{\circ} 25$.

DU BELLAY, Joachim (2007). La Deffence et illustration de la langue françoyse; et l'Olive. Genève : Droz, « Textes littéraires français ».

FRAGONARD, Marie-Madeleine, SCHRENCK, Gilbert (2000). Joyeusement vivre et honnestement penser. Mélanges offerts à Madeleine Lazard, choix d'articles réunis par Marie-Madeleine Fragonard et Gilbert Schrenck. Paris : Honoré Champion, « Colloques, congrès et conférences sur la Renaissance », n $\circ 17$.

GABIANI, Vincenzo. I Gelosi (1606). MF 7027, General Microfilm Company, « Italian books. 1601-1700», Watertown.

GOYET, Francis (1990). Traités de poétique et de rhétorique de la Renaissance. Paris, Librairie Générale française, « Le Livre de Poche ", n 6720.

GRAZZINI, Anton Francesco (1750). La Gelosia, dans : Del teatro comico fiorentino, t. III. Florence.

LANGLET, Irène (2003). Le Recueil littéraire. Pratiques et théories d'une forme. Rennes: Presses Universitaires de Rennes, «Interférences ».

LARIVEY, Pierre de (1855). Notes d'Eugène-Emmanuel Viollet-le-Duc. Ancien Théâtre François ou collection des ouvrages dramatiques les plus remarquables depuis les Mystères jusqu'à Corneille [1579], t. V et VI. Paris : « Bibliothèque elzévirienne ».

LARIVEY, Pierre de (2011). Théâtre complet. Les Six Premières Comédies facécieuses (Le Laquais, La Vefve, Les Esprits) t. I [1579], édition critique de Luigia Zilli. Paris : Classiques Garnier, « Bibliothèque du théâtre français ».

LAZARD, Madeleine (1980). Le Théâtre en France au XVI siècle. Paris : Presses Universitaires de France, « Littératures modernes ».

MAZOUER, Charles (2002). Le Théâtre français de la Renaissance. Paris : Honoré Champion,

« Dictionnaires et références ", $n^{\circ} 7$.

MÉDICIS, Lorenzino de (1912). L'Aridosia, dans : Ireneo Sanesi, Commedie del Cinquecento, t. II, G. Laterza, Bari, « Scrittori d'Italia », n 25, 1912.

MONFERRAN, Jean-Charles (2011). L'École des Muses. Les arts poétiques français à la Renaissance

(1548-1610). Sébillet, Du Bellay, Peletier et les autres. Genève : Droz, « Les Seuils de la modernité », vol. 12 .

RAzZI, Girolamo (1565). La Cecca. Venise : appresso Andrea Ravenoldo.

\section{RÉSUMÉS}

Publié en 1579, le recueil des Six Premières Comédies facécieuses de Pierre de Larivey consacre son auteur comme le plus prolifique dramaturge comique $\mathrm{du} \mathrm{XVI}^{\mathrm{e}}$ siècle. Se targuant dès l'épître dédicatoire du caractère profondément «nouveau " de son ouvrage, il se dissimule pourtant derrière ces affirmations de primeur et d'innovation une tout autre genèse de l'œuvre. Une lecture parallèle avec le théâtre italien révèle en effet le corpus exclusivement transalpin à l'origine de l'ensemble de ses pièces. Cette «nouveauté » renaissante n'est pas tant celle de l'originalité de l'auteur que celle d'une recréation en langue française de ces six pièces. Il s'agit ainsi d'identifier au fil de notre étude les divers phénomènes d'imitation à l'œuvre dans ce 
recueil comique pour saisir la subtilité du travail d'appropriation culturelle mis en place par Larivey.

Published in 1579, the collection of the Six Premières Comédies facécieuses by Pierre de Larivey sets him as the most prolific comic playwright of the $16^{\text {th }}$ century. However, while he claims the deeply innovative status of his work in the dedicatory epistle, a whole different genesis hides itself behind these declarations of novelty and newness. A parallel reading with the Italian theatre reveals indeed the Italian origins of these plays. This humanist "novelty" refers not so much to the author's originality than to the French rewriting of these plays. That is why we strive to identify through this research the diversity of imitation's manifestations in this literary collection of comedies, in order to understand the subtlety of Larivey's work of cultural appropriation.

INDEX

Keywords : Larivey (Pierre de), imitation, Renaissance, erudite comedy, collection

Mots-clés : Larivey (Pierre de), imitation, Renaissance, comédie érudite, recueil

\section{AUTEUR}

\section{ANNE ROGÉ}

Université de Rennes II. Université de Notre Dameanne.roge0[at]gmail.com 